\title{
Mouth Breathing-Its Consequences, Diagnosis \& Treatment
}

\section{Sukhdeep Singh ${ }^{1}$, Neha Awasthi ${ }^{2}$ and Taru Gupta ${ }^{3 *}$}

${ }^{2}$ Professor \& Head of Department, Department of Pediatric and Preventive

Dentistry, School of Dental Sciences, Sharda University, Greater Noida, Uttar Pradesh, India

${ }^{2}$ Reader, Department of Pediatric and Preventive Dentistry, School of Dental Sciences, Sharda University, Greater Noida, Uttar Pradesh, India

${ }^{3}$ Postgraduate Student, Department of Pediatric and Preventive Dentistry, School of Dental Sciences, Sharda University, Greater Noida, Uttar Pradesh, India

*Corresponding Author: Taru Gupta, Postgraduate Student, Department of Pediatric and Preventive Dentistry, School of Dental Sciences, Sharda University, Greater Noida, Uttar Pradesh, India.
Received: March 14, 2020

Published: April 22, 2020

(C) All rights are reserved by Taru Gupta., et al.

\section{Abstract}

The mouth breather is a patient with multiple conditions and therefore requires comprehensive and multidisciplinary clinical look to early detect this syndrome, minimizing its deleterious effects. A pediatric dentist may be one of the first healthcare professionals to come in contact with a patient who exhibits mouth breathing and thus it is important to have a sound knowledge to perform correct diagnosis and effective treatment. A review of current data on the skeletofacial, dental and gingival changes that occur in mouth breathing individuals is given, with the intention of raising the awareness of dental professionals towards the special needs of these patients.

Keywords: Mouth Breathing; Malocclusion; Developing Dentition; Dentofacial

\section{Introduction}

The prime physiological functions of the oral cavity are respiration, suckling, swallowing, mastication and speech. All these functions are balanced; the disturbance in one oral function results to an abnormal growth and development of bony and soft tissue structures of the craniofacial complex [1].

Mouth breathing refers to the state of inhaling and exhaling through the mouth. The literature describes the prevalence of mouth breathing as ranging from 5 to $75 \%$ of tested children. The mouth does not usually contribute in respiration. Increased struggle to the flow of air through the nasal passage may be considered to be the key reason of mouth breathing [2].

The reduced nasal respiratory function causes altered tongue posture which result in inferior positioning of mandible thereby inducing changes in neck and facial muscular activity leading to development of dental and facial characteristics [3].

Mouth breathing has been declared to have serious effects on the growth of the facial skeleton and occlusion of teeth on account of the displacement of normal lateral, buccal and lingual muscular forces [4].
Bresolin D., et al. evaluated allergic mouth-breathing subjects and concluded that mouth breathers had longer faces [5], Tourne LP concluded that the switch from a nasal to an oronasal breathing pattern induces functional adaptations that include an increase in total anterior face height and vertical development of the lower anterior face [6].

Faria PT., et al. indicated that changed mode of breathing was associated with maxillo-mandibular retrusion in relation to the cranial base in the mouth breathers and the SNGoGn (Sella-Nasion to Gonion-Gnathion) and NSGn (Nasion-Sella to Gnathion) angles were greater in the mouth-breathing group [7].

Sassouni determined mouth breathing as a habitual respiration through the mouth instead of the nose [8]. Mouth-breathing is a substitute respiratory mode and consists of a mechanically incorrect form of respiration. It has a multifactor etiology and multiple consequences. Mouth breathing is a deleterious habit [9].

Due to its range of co-morbidities, mouth breathing (MB) has been a concern for healthcare professionals in various areas. Mouth breathing (MB) is also an etiological factor for sleep- disordered breathing (SDB) during childhood. Both habit and obstruction may cause facial muscle imbalance and craniofacial changes [2]. 
In mouth breathers, the carbon dioxide levels decrease in lungs and blood fall, and this leads to lower oxygen supply of the body cell [10]. Oral respiration, low tongue posture and elongation of lower anterior facial height are apparent at 3 years of age but more commonly detected after age five [11].

General and pediatric dentists may be in the best position to screen and treat patients who suffer from upper airway obstruction/mouth breathing. Dentists usually see patients on a regular basis every six months, and swollen tonsils can be easily detected by using a mouth mirror to look at the back of the patient's throat. All patients whether children, adolescents, and adults should be screened for upper airway obstruction. Mouth breathing is best managed by using a multidisciplinary approach involving pediatricians, physicians, dentists, and ear-nose-throat (ENT) specialists [12].

\section{Definitions}

Mouth breathing is a deleterious habit. It may be determined as an oral habit in the cases when there is no anatomical factors and obstacle for nose breathing [13].

C. V. Tomes introduced in 1872 the term "adenoid facies and determined typical dentofacial appearance in patients with nasal breathing difficulties. The face becomes long and narrow [10].

Open bite, hyperdivergent growth pattern, proclined upper incisors, increased lower facial height, steeper mandibular plane angle, lowering of the chin and increase in the gonial angle are among these features.

A little later, in 1889, Kingsley undertook another study, according to which we have a normal skeletal formation and development in children with severe nose breathing difficulties. Similar researches are aiming to compare the dentofacial development of mouth breathers towards nose breathing patients and have been carried out by other scientists too [14].

In Chacker's opinion, nasal breathing difficulties are defined as a long or prolonged exposition of the frontal facial tissues to the drying up effect of the inhaled air [8].

Sassouni determined mouth breathing as a habitual respiration through the mouth instead of the nose [8].

A little later, Merle offered the term oronasal breathing instead of oral or mouth breathing as he considers this term is more correct and exact [8].
Nowadays the viewpoint is adopted that mouth breathers are those people who breathe through the mouth even when at rest. They should be distinguished from nasal breathers who breathe through the mouth only upon intensive physical loads but through the nose at rest [15].

\section{Classification}

Sim and Finn (1987) classified mouth breathers into the following three categories according to the etiology as [16]:

- Obstructive: Children who have an increased resistance to or a complete obstruction of the normal flow of air through the nasal passages. The child is forced by sheer necessity to breathe through the mouth. Seen in ectomorphous individuals with long narrow $\mathrm{f}$

- $\quad$ aces and nasopharyngeal passages.

- Habitual: Habitual mouth breather is a child who continually breathes through his mouth by force of habit, although the abnormal obstruction has been removed.

- Anatomical: The anatomic mouth breather is the one whose short upper lip does not permit closure without undue effort.

- Total blockage: Nasal passages are completely blocked.

- $\quad$ Partial blockage (Figure 1).
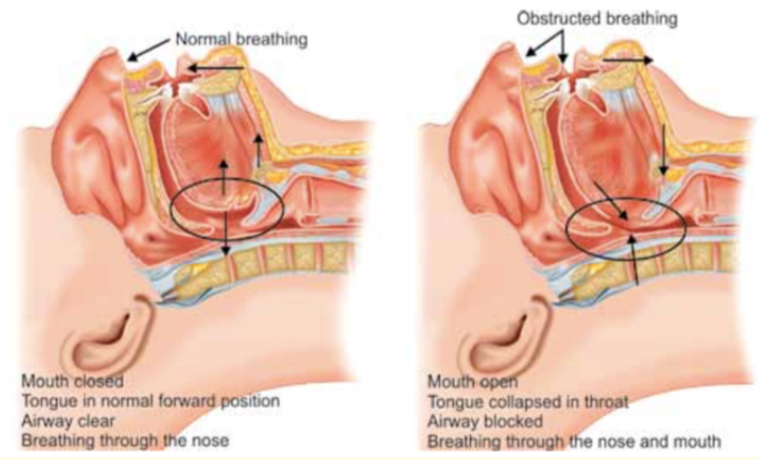

Figure 1: Difference between normal and obstructed breathing.

\section{Etiology}

The etiology of mouth breathing is multifactorial. Mouth breathing is majorly caused due to nasal obstruction. Nasal obstruction can result from either congenital or postnatal causes and may amplify resistance to air-flow and impair sucking-swallowing responses, with increased risks of aspiration or of more severe and 
threatening respiratory distress conditions. In addition, nasal obstruction alters the "trophic" flow of sensory information towards the olfactory brain [17].

\section{Nasal obstruction $[7,16]$ :}

- $\quad$ Enlarged turbinate: This may be due to: allergies, chronic infections of mucous membrane, atrophic rhinitis, hot and dry climatic conditions, polluted air.

- Hypertrophy of pharyngeal lymphoid tissue (adenoids): Repeated infection results in the over growth of lymphoid masses blocks the posterior nares, rendering mouth breathing necessary.

- Intranasal defects: Deviated nasal septum, subluxation of septum, thickness of septum, bony spurs, polyps.

- Allergic rhinitis Continuous infections and toxins of the bacteria may sensitize the tissue to develop allergic reactions.

The most common cause of mouth breathing is the presence of obstacles in the nasopharyngeal region, which increases nasal resistance. The most extreme forms are due to congenital laryngomalacia, bilateral choanal atresia, or oronasal defects associated with Pierre Robin syndrome. Less extreme forms involve choanal stenosis, unilateral choanal atresia, or defects of the nasal septum related to cleft palate.

Other mechanical causes such as those due to obstructive tissue masses (adenoid or/and tonsillar hypertrophy) prevail during later development. More benign, short-term obstructive forms derive from mucosal accumulation due to neonatal infections or allergic rhinitis [17]. Intranasal defects such as deviated nasal septum, bony spurs or polyps may also cause mouth breathing [8].

\section{Consequences of mouth breathing}

There are different general mechanisms which may explain the consequences of mouth breathing. They are mainly composed of biochemical, physiological, immunological and anatomical disturbances which has been attributed to mouth breathing. Among the biochemical and physiological disorders are the lower oxygen absorption (chronic hypoxemia), increased $\mathrm{CO}_{2}$ concentration (hypercapnia), and its related changes in the acid-base balance, towards respiratory acidosis. Also, there is increased water and energy loss, and changes in salivary profile. It has been shown that healthy subjects, experience a $42 \%$ increase in net water loss when they switch the breathing mode from nasal to oral expiration during tidal breathing. The authors considered that increased water and energy loss by oral breathing could be a contributing factor to the symptoms seen in patients suffering from nasal obstruction [18].
Also a decreased nocturnal growth hormone (GH) release has been reported. It has been considered that snore at night and struggle for air interferes with growth hormone production in some way [19].

Inflammatory and oxidative mediators release had also been linked to mouth breathing, however it is not clear if they are the result of mouth breathing, atopia, other related disturbances or the complex interactions among then. The oxidant nitric oxide (NO) is produced by the action of the enzyme NO synthase (NOS) on Larginine in different cell types and found in air exhaled by humans. Most of the exhaled NO is derived from the upper airways and increases in patients with untreated asthma and allergic rhinitis, diseases which exhibit mutual interactions with mouth breathing. The induction of iNOS (inducible NOS) in patients with allergic rhinitis increases nasal NO which in turn produces the symptoms of nasal obstruction and rhinorrhoea, contributing to mouth breathing [20].

With respect to the immune system, lowered response has been described which is linked to poor health. It has been stated that nasal breathing produces a tissue hormone that regulates normal blood circulation. It also filters, warms and moisturizes the air. The lack of oxygen in mouth breathers is considered to weaken the immune system [21].

Anatomical effects of mouth breathing are the result of the adaptation of the growing tissues to the abnormal breathing profile. They include among others, large load on the upper back and neck muscles and deformity in the airway passage which are involved in postural disturbances and obstructive sleep apnea. As the jaw in mouth breathers is positioned too far back, along with the tongue, the upper airway is constricted. Enlarged tonsils and adenoids due to chronic allergies may be the primary cause for mouth breathing; however, mouth breathing will also cause a further increase in tonsil size, constricting the airway and posing obstacles to nasal breathing. The open mouth will also cause a decreased contractile efficiency of the upper airway muscles affecting nasal breathing [22]. Also, dentofacial structures' growing is affected leading to altered profile and malocclusion, among others [21].

Oral conditions related to mouth breathing- Dry mouth and lips, Dental caries, Periodontal disease, Secondary halitosis, Craniofacial deformity and malocclusion, Abnormal swallowing.

Medical conditions related to mouth breathing-Altered head, neck and body posture, Obstructive sleep apnea (OSA), Poor physical and learning performance, Asthma.

\section{Clinical features of mouth breathing patients}

Mouth breathing patients have characteristic intraoral and extraoral symptoms. In the period between 1970 and 1980, Linder- 
Aronson reported the connection between mouth breathing and craniofacial appearance including long face, anterior open bite, overjet, posterior crossbite [23].

\section{Effect on face [16] (Figure 2):}

1. Lips slack and stay open

2. Short upper lip

3. Molding action of upper lip on incisors is lost thereby resulting in proclination and spacing.

4. Lower lip: heavy and everted.

5. Tongue is suspended between upper and lower arches resulting in constriction of buccal segment.

6. Increased mandibular plane angle.

7. Retrognathic maxilla and mandible.

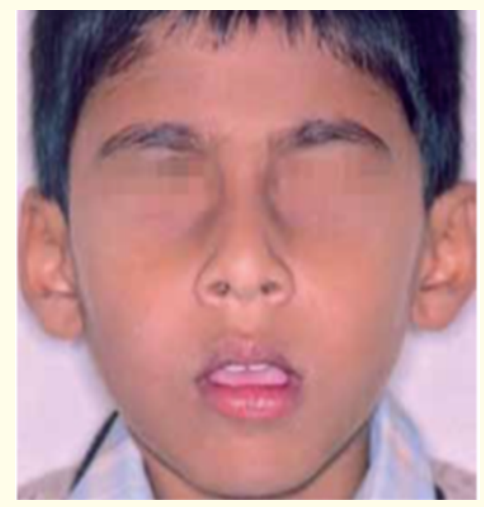

Figure 2: Adenoid facies.

\section{Effect on occlusion of teeth [16]}

1. Proclination of anteriors justified by interposition of the hypertonic lower lip between maxillary and mandibular incisors provoking labioversion of the maxillary incisors. Koski reported that the mandibular incisors presented retroclination in relation to the mandibular plane in patients with hypertrophic adenoid [4].

2. Distal relation of mandible to maxilla.

3. Lower anteriors supraerupt to touch the palatal tissues.

4. Posterior crossbite.

5. Anterior open bite.

The maxilla and mandible were more retrognathic in the mouth breathing group. The maxilla were more retrognathic owing to upper airway obstruction resulting from the hypoplasia of the maxillary sinus and narrowing of the nasal cavities. Narrow palatal and cranial widths are also associated. This is due to the low set position of the tongue in order to allow an adequate inflow of air through the mouth.

Thus, an imbalance of forces exerted by the tongue and facial musculature on the maxilla leads to a constricted maxillary arch. There may be flaring of incisors and a decrease in the vertical overlap of the anterior teeth [24] (Figure 3).

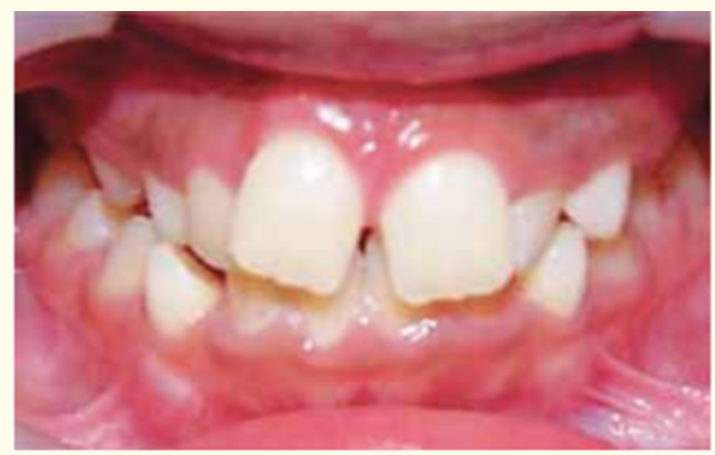

Figure 3: Effects of mouth breathing on teeth.

Effect on gingiva: Gingival tissues: Constant wetting and drying of the gingiva causes irritation, saliva about the exposed gingiva tends to accumulate debris resulting in an increase in bacterial population [16].

Effect on speech: Abnormalities of the oral and nasal structures can seriously compromise speech performances. Nasal tone in voice is seen [24].

Effect on lip: These patients frequently have a lip apart posture, although the lip apart posture should not be regarded as pathognomonic for nasal obstruction. On smiling, many of these reveal large amounts of gingiva producing the 'gummy smile'. Children who breathes through mouth have a short thick incompetent upper lip and a voluminous curled over lower lip [24] (Figure 4).

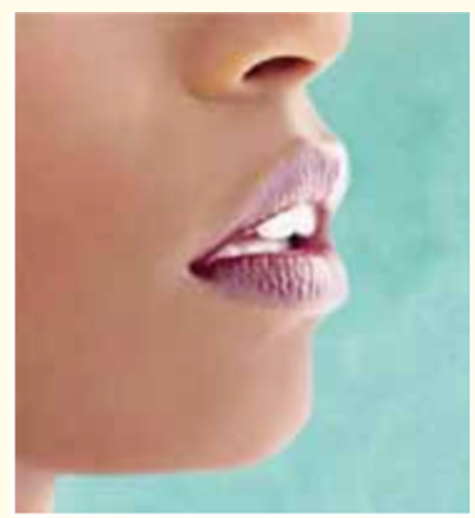

Figure 4: Lip apart swallow. 
Effect on external nares: Long-standing nasal airway obstruction can lead to a disuse atrophy of the lateral cartilage. The result is a slit-like external nares with a narrow nose [24].

Other effects: It may lead to otitis media. The palatoglossus muscle is active in the case of nose breathers, whereas the levator palatine activity is lower when nose breathing was compared with mouth breathing. There is also a dull sense of smell and loss of taste. The occurrence of halitosis was high among the children with mouth breathing.

Mouth breathing irritates the mucosa, and these children often will have swollen tonsils and adenoids, one of the major causes of upper airway obstruction, sleep disorders and sleep apnea [5] (Figure 5).
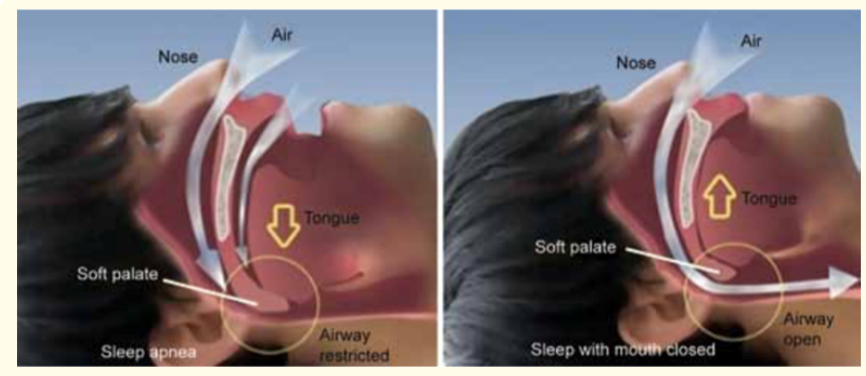

Figure 5: Sleep apnea.

According to Subtelny, the deep palate in mouth breathers is an illusion due to the upper jaw compression. Brodie thinks that jaw compression is due to an imbalance between cheek muscles and tongue muscles. As the pressure in the area of premolars and molars is bigger than this in the area of canines, the changes in the posterior region are greater [10].

Angle, Brodie, Hawking and Moyers accept the role of muscles in upper jaw formation, while other authors like Brash, Linder-Aronson and Backstrom disclaim this theory.

Angle and Hawking do not find a deviation in the dental arch form in mouth breathing children versus such breathing through the nose. As opposed to this, many authors find crooked frontal teeth more often in upper jaw [10].

The studies confirm that class II malocclusion is more often and related with mouth breathing patients [25]. Angle, Huber and Reynolds and Moyers report that mouth breathing has an impact on the development of all classes of malocclusions [26].

In contrast to the above indicated, Howard examine a lot of mouth breathing children who are with class I molar relationship.
According to Rakosi and Schilli mouth breathing plays a role in aetiology and pathogenesis of class III malocclusion [27]. A notinsubstantial percentage of mouth breathing children have a deep bite. It is most often due to the distal position of the lower jaw and lack of incisor contact. Hawkins and Rachel found that deep bite is due to underdevelopment of premolars and molars segments [10].

\section{Diagnosis}

An early diagnosis is crucial for the correction of mouth breathing and to avoid any associated conditions. Correctly diagnosing a habit of mouth breathing may require a detailed case history, clinical examination and diagnostic tests.

A detailed case history regarding the development of the habit, duration, frequency and associated symptoms must be recorded. This should be followed by a clinical examination of the intraoral and extraoral features of the patient and making a record of the same [2].

It is important to fully diagnose a patient with the habit of mouth breathing through various clinical tests available. The mirror test and the water retention test are among the breathing tests most cited in the literature. The lip seal test is the most frequently used, followed by the mirror test and the water retention test [28].

Diagnosis of mouth breathing is made on the basis of patient history and clinical examination. Patients are asked whether, in their opinion, they were mouth breathers, and also whether they had dry mouth on awakening. The examination comprised observation coupled with various diagnostic tests for mouth breathing. Patient reaction is observed when one of the nostrils is closed and the lips sealed. Nose breathers usually demonstrate good control of the alar muscle, and this is lacking in mouth breathers.

Also, the following diagnostic tests are performed to confirm the diagnosis of mouth breathing [29].

Mirror test: The mirror test is also called the fog test. A doublesided mirror is held between the nose and the mouth. Fogging on the nasal side of the mirror indicates nasal breathing while fogging on the oral side-mouth breathing.

Massler's water holding test: Patient is asked to hold the mouth full of water. Mouth breathers cannot retain the water for a long time [16].

Massler and zwemer butterfly test/cotton test: Butterfly shaped cotton strands are placed over the upper lip below nostrils. On exhalation if the fibres flutter downwards, the patient is a nasal breather and if the fibres flutter upwards, the patient is a mouth breather [16]. 
Inductive plethysmography (Rhinometry): The total airflow through the nose and mouth can be quantified using inductive plethysmography, the only reliable way. This allows the percentage of nasal and oral respiration to be calculated. A minority of the long face children had less than $40 \%$ nasal breathing. Nasal air flow characteristics are studied by using devices consisting of flow meters and pressure gauges [16].

One cross-sectional study used the plethysmograph on normal children and reported: Prior to age 8- there were as many oral or predominantly oral breathers as nasal or predominantly nasal breathers. After age 8-the majority of the children were nasal or predominantly nasal breathers (Warren., et al. 1990).

Cephalometrics: Can be used to calculate amount of nasopharyngeal space, size of adenoids and to know he skeletal patterns of the patient by taking various cephalometric angles $[4,26]$.

Assessment of nasal function: The adequacy of nasal breathing was assessed by asking the children to breathe through their nose for 1 minute after putting water in their mouth and by fogging or condensation on mirror which was placed both near nose and mouth simultaneously and referred to the ENT Department where a detailed clinical and physical examination was done. Following which a PA view nasopharynx radiograph was taken to examine the presence of adenoids. The presence of adenoid hypertrophy was confirmed using examination by direct fiberoptic nasopharyngoscopy [11].

Assessment of dentofacial changes: The subjects were made to stand in the cephalostat (rotagraph plus) with the Frankfort Horizontal plane parallel to the floor and teeth in centric occlusion. Agfa digital X-ray film ( $8^{\prime \prime} \times 10^{\prime \prime}$; speed E) were exposed at $72 \mathrm{kVp}$, $10 \mathrm{~mA}$ for $0.8 \mathrm{~s}$ from a fixed distance of 60 inches following the standard technique [11].

Cephalometric assessment was made by means of a combination of manual and computerized methods. The various linear and angular dental measurements and soft tissue measurements were recorded as nasal breathers (NB) and mouth breathers (MBA and MB) for comparison with cephalometric variables of a normal child [21].

There are objective anatomical characteristics which show the presence of a difficult nasal breathing. The size of tonsils (Figure 6) is also determined during the clinical intraoral examination [10].

The widely adopted at a global scale Friedman score is used for assessment of the obstruction stage on level mesopharynx on the part of the tongue. It is a modification of the Mallampati classifica-
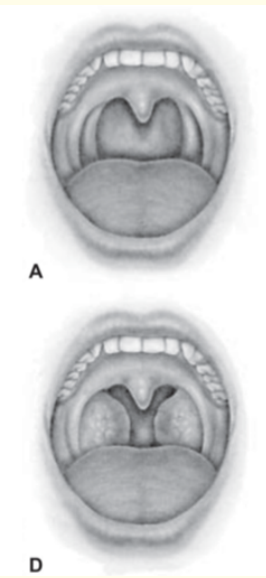
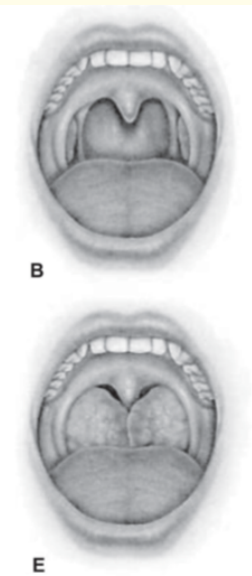

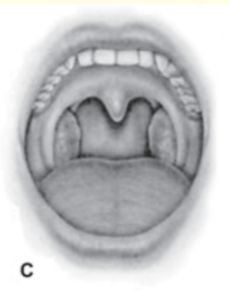

Figure 6: Illustration of tonsil grades.

A. TS 0: Status post tonsillectomy; B. TS 1: Tonsils are behind the anterior palatal arches, the posterior and lateral walls of mesopharynx are accessible for examination; C. TS 2: Tonsils placed medially out of the arches but do not cover the lateral columns; D. TS 3: Tonsils cover the lateral columns but do not reach the midline; E. TS 4: Tonsils touch each other in the midline, the so called "kissing tonsils".

tion upon which the tongue is in the oral cavity during the examination [10] (Figure 7).

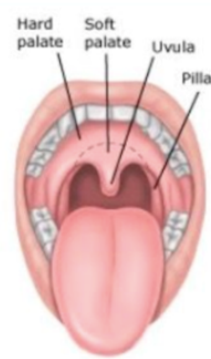

Class I

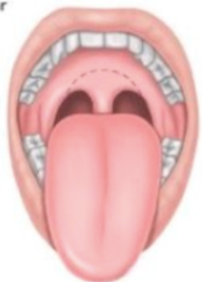

Class II

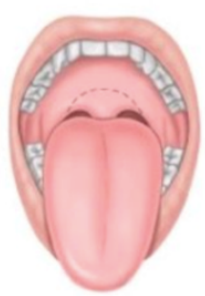

Class III

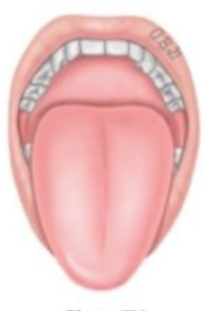

Class IV
Figure 7: Graphic representation of

(A) Mallampati score classes.

A: Class I: When tongue is in the oral cavity, tonsils, lateral columns and the whole uvula are easy to examine; B: Class II-The whole soft palate and the uvula are visible, excluding palatal tonsils; C: Class III-A part of the soft palate is visible but not the whole uvula; Class IV: The soft palate is visible only.

Treatment of mouth breathing Treatment consideration

- Age of child: Mouth breathing in many instances is self-correcting after puberty. This can be attributed to the increase 
in nasal passages as the child grows, thereby relieving the obstruction caused due to enlarged adenoids.

- ENT examination: An otorhinolaryngologist examination may be advised to determine whether conditions requiring treatment are present in the tonsils, adenoids or nasal septum. If habit continues after removal of cause then it is habitual.

- Prevention and interception: Mouth breathing can be intercepted by use of an oral screen [16,24].

Treatment according to symptoms

Gingiva of the mouth breathers should be restored to normal health by coating the gingiva with petroleum jelly.

It may be divided under three main factors:

1. Remove the cause: Etiological agents should be treated first. Removal of nasal or pharyngeal obstruction by surgery or local medication should be sought. If a respiratory allergy is present, it should be brought under control.

2. Rapid maxillary expansion has been reported to reduce the rapid maxillary expansion.

3. Intercept the habit: If the habit continues even after the removal of the obstruction then it should be corrected.

\section{Methods of correction:}

\section{a. Exercises:}

- During day time-hold pencil between the lips.

- During night time-tape the lips together with surgical tape in habitual mouth breathing

- Hold a sheet of paper between the lips.

- Piece of card $1 \times 11 / 2$ " held between the lips.

- $\quad$ Patients with short hypotonic upper lip: Stretch the upper lip to maintain lip seal or stretch in downward direction toward the chin.

- Button pull exercise: A button of 11/2" diameter is taken and a thread is passed through the button hold. The patient is asked to place the button behind the lip and pull the thread, while restricting it from being pulled out by using lip pressure.

- Tug of war exercise: This involves two buttons, with one placed behind the lips while the other button is held by another person to pull the thread. Blow under the upper lip and hold under tension to a slow count of 4 repeat 25 times a day. Draw upper lip over the upper incisors and hold under tension for a count of 10 . b. Maxillothorax myotherapy: This was advocated by Macaray 1960. These expanding exercises are used in conjunction with the Macaray activator. Macaray constructed an activator out of aluminum with which development of the dental arches and dental base relationship could be corrected at the same time as encouraging mouth breathing. The mouth breather holds the activator in the mouth and at the same time with the left and right arms alternately carries out 10 exercises 3 times a day $[16,24]$.

c. Oral screen: First introduced by Newell in 1912.It is a myofunctional appliance that is easy to fabricate and easy to wear. It works on the principle of both force application and force elimination $[16,24]$.

A new treatment protocol given by Denotti., et al. in 2014 includes a rapid palatal expander bonded on the deciduous molars, in association with the use of silicone myofunctional devices such as the nasal stimulator and the oral obturator, that speeds and increases the effectiveness and stability of treatment [30].

\section{Definition}

- Graber: An appliance that utilizes the musculature to control abnormal muscle habits and aids in correction of the developing malocclusion [31].

- C Phillip Adams: A removable appliance, used to deflect or eliminate muscle forces on certain teeth. Commonly placed in the vestibule between the lips, cheeks and the teeth.

- Principle: It is a functional appliance by virtue of the fact that it produces its effects redirecting the pressures of the muscular and soft-tissue curtain of the cheeks and lips. It works on the principle of both force application and force elimination. For example, anterior teeth proclination can be corrected utilizing the principle of force application. The screen comes in contact with the proclined teeth so that the forces from the lips are transmitted directly to the proclined teeth through the screen. Posterior cross bite can be corrected utilizing the principle of force elimination by providing a spacer between the teeth and the screen [32].

Indication

- Habit-correcting appliance.

- It helps retrain and strengthen lip action.

- $\quad$ Lip exercises are possible with oral screen, which improves the tonicity of the lips.

- To correct simple labioversion of the maxillary anterior teeth. 
Contraindication: It should not be used if the child has nasorespiratory distress or a nasal obstruction [31].

Construction: Upper and lower impressions are made must reproduce the full depth of the labial sulcus and casts are prepared. The casts are occluded and sealed. Posteriorly, the appliance will extend up to the distal margin of the last erupted teeth. The upper and lower borders will extend to the full depth of the sulcus.

Rapid maxillary expansion (RME): Patients with narrow, constricted maxillary arches benefit from RME procedures aimed at widening of the arch. It increases nasal air flow and decrease nasal air resistance. Increase in intranasal space occurs due to outer walls of nasal cavity moving apart[16].

\section{Correction of malocclusion}

- Children with class I skeletal and dental occlusion and anterior spacing oral shield appliance.

- Class II division 1 without crowding, age 5 to 9 years, monobloc activator both to correct malocclusion and deterrence of habit.

- Class III malocclusion: interceptive methods are recommended as chin cap [16].

\section{Discussion}

Mouth breathing is a potential etiological factor for the alterations of the occlusion and normal facial growth. When combined with other parafunctional habits, the harm to the stomatognathic system is even greater. According to Cattoni., et al. (2007) [33], children with this habit exhibit pathologic adaptations regarding postural and morphological characteristics of the stomatognathic system. Thus, the early diagnosis of mouth breathing and proper intervention are suggested to avoid the emergence of orofacial abnormalities.

Mouth breathing habit was the second most prevalent habit in the study conducted by Deepak P Bhayya and Tarulatha R Shyagali [34] with the incidence rate of $17 \%$. This incidence was higher when compared to the findings of the previous studies. Amr AbouEI-Ezz., et al. in their study on prevalence of mouth breathing habit and its probability as etiological factor of malocclusion have concluded that malocclusion is highly associated with habits existence and this relationship is statistically highly significant $(\mathrm{p}<0.001)$. Motta LJ [35] finds out that there were a significantly greater number of boys with the mouth breathing pattern than girls. Guaba., et al. [36] and Kharbanda., et al. [37] reported tongue thrusting and mouth breathing as the most prevalent habits in their studies.

Malhotra S., et al. found out that children who breathe predominantly through their mouth pose difficult problems for healthcare professionals. It was also found that all subjects with mouth- breathing habit exhibited significant increase in facial height, mandibular plane angle and gonial angle [1]. Conti., et al. observed that Postural problems were significantly more common among children in the group with mouth breathing syndrome, highlighting the need for early interdisciplinary treatment of this syndrome [38].

Bresolin., et al. found that mouth breathers had longer faces with a narrower maxilla and retrognathic jaws [39] and Trask found that allergic children who were mouth breathers had longer and more retrusive faces than nasal breather children [40].

The mouth breather is a patient with multiple conditions and therefore requires comprehensive and multidisciplinary clinical look to early detect this syndrome, minimizing its deleterious effects.

\section{Conclusion}

The habit to breathe through the mouth instead of the nose is an exclusively topical problem nowadays. Despite the development of medicine and contemporary early diagnostic tools, the percentage of mouth breathing children grows. The clinical symptoms are diverse in respect of severity depending on mouth breathing duration. If not treated developmental deficiencies progress and thereby the severity of structural changes in the body rises. Mouth breathing impacts - facial development, alignment, functioning and growth of the body.

Dentist often diagnose such patients with anterior open bite, an overjet, a distal position of the lower jaw, a narrowing of the upper jaw and an increase in the height of the lower facial third [10].

Thus, this led to the conclusion that all people with mouthbreathing habit exhibited significant lower incisor proclination, lip incompetency and convex facial profile. The presence of adenoids accentuated the facial convexity and mentolabial sulcus depth [12].

A multidisciplinary team should work to have early diagnosis and appropriate treatment, preventing the consequent disorders of chronic mouth breathing. Because upper airway obstruction is an obstacle to normal dentofacial development, mouth breathing children deserve prompt attention before growth has proceeded irreversibly. The early recognition of such facial patterns may be utilized to identify those breathing compromised individuals who are likely to develop such types of malocclusions [12].

Hence a joint effort by pedodontist, orthodontist, otorhinolaryngologist and pediatrician is thus required for reducing continuing detrimental effects of breathing impairments on facial characteristics [1]. 


\section{Bibliography}

1. Malhotra S., et al. "The effect of mouth breathing on dentofacial morphology of growing child". Indian Society of Pedodontics and Preventive Dentistry 30.1 (2012): 27-31.

2. Nilufer Nadaf., et al. "Mouth Breathing- A Harmful Habit in a Young Child". ARC Journal of Forensic Science 3.2 (2018): 2529.

3. Klein JC. "Nasal respiratory function and craniofacial growth". Archives of Otolaryngology - Head and Neck Surgery 112.8 (1986): 843-849.

4. Paul JL and Nanda RS. "Effect of mouth breathing on dental occlusion”. The Angle Orthodontist 43.2 (1973): 201-206.

5. Bresolin D., et al. "Mouth breathing in allergic children and its relationship to dentofacial development". American Journal of Orthodontics 83 (1983): 334-340.

6. Tourne LP. "The long face syndrome and impairment of the nasopharyngeal airway". The Angle Orthodontist 60 (1990): 7-76.

7. Faria PT., et al. "Dentofacial morphology of mouth breathing children". Brazilian Dental Journal 13 (2002): 129-132.

8. Bishara SE. "Textbook of Orthodontics". Philadelphia, WB Saunders Co (2001): 606.

9. Milanesi JD., et al. "Childhood mouth- breathing consequences at adult age: ventilatory function and quality of life". Fisioterapia em Movimento 27.2 (2014): 211-218.

10. Valcheva Z., et al. "The role of mouth breathing on dentition development and formation". Journal of IMAB 24.1 (2018): 1878-1882.

11. Basheer B., et al. "Influence of mouth breathing on the dentofacial growth of children: A cephalometric study". Journal of International Oral Health 6.6 (2014): 50-55.

12. Jefferson Y. "Mouth breathing: adverse effects on facial growth, health, academics, and behavior". General Dentistry 58.1 (2010): 18-25.

13. Arathi Rao. "Principle and Practice of Pedodontics". $3^{\text {rd }}$ edition. New Delhi (2012): 169-170.

14. Gill DS and Naini FB. "Orthodontics: Principles and Practice". Wiley-Blackwell (2011): 281.
15. Souki BQ., et al. "Prevalence of malocclusion among mouth breathing children: do expectations meet reality?" International Journal of Pediatric Otorhinolaryngology 73.5 (2009): 767- 773 .

16. Singh G. "Textbook of Orthodontics". $2^{\text {nd }}$ edition. Chapter 49oral habits and their management: 581-612.

17. Trabalon M and Schaal B. "It takes a mouth to eat and a nose to breathe: abnormal oral respiration affects neonates' oral competence and systemic adaptation". International Journal of Pediatrics (2012).

18. Svensson S. "Increased net water loss by oral compared to nasal expiration in healthy subjects". Rhinology 44.1 (2006): 74-77.

19. Peltomäki T. "The effect of mode of breathing on craniofacial growth-revisited”. European Journal of Orthodontics 29.5 (2007): 426-429.

20. Kent DT and Soose RJ. "Environmental factors that can affect sleep and breathing: allergies". Clinics in Chest Medicine 35.3 (2014): 589-601.

21. Triana BE., et al. "Mouth breathing and its relationship to some oral and medical conditions: physiopathological mechanisms involved". Revista Habanera de Ciencias Médicas 15.2 (2016).

22. Margosian Conti PB., et al. "Assessment of the body posture of mouth-breathing children and adolescents". The Journal of Pediatrics 87.4 (2011): 357-363.

23. Linder-Aronson S. "Effects of adenoidectomy on dentition and nasopharynx". American Journal of Orthodontics 65.1 (1974): 1-15.

24. Tandon S. "Textbook of Pedodontics". $2^{\text {nd }}$ Edition. Chapter 39: Commonly Occurring Oral Habits in Children and their Management: 492-526.

25. Joshi MR. "Study of dental occlusion in nasal and pro-nasal breathers in Maharashtrian children". J All India D.A. 36 (1964): 247-249.

26. Huber RE and Reynolds JW. "A dentofacial study of male students at the University of Michigan in the physical hardening program". American Journal of Orthodontics and Oral Surgery 32 (1946): 1-21. 
27. Rakosi T and Schilli W. "Class III anomalies: a coordinated approach to skeletal, dental, and soft tissue problems". Journal of Oral and Maxillofacial Surgery 39.11 (1981): 860-870.

28. Pacheco MC., et al. "Guidelines proposal for clinical recognition of mouth breathing children". Dental Press Journal of Orthodontics 20.4 (2015): 39-44.

29. Bhatia Anu and Sharma., et al. "A randomized clinical trial of salivary substitute as an adjunct to scaling and root planing for management of periodontal inflammation in mouth breathing patients". Journal of Oral Science 57 (2015): 241-247.

30. Denotti G., et al. "Oral breathing: new early treatment protocol". Journal of Pediatric and Neonatal Individualized Medicine 3.1 (2014): e03 0108.

31. Graber TM., et al. "Dentofacial orthopedics with functional appliances" (1985): 496-519.

32. Knosel M., et al. "A controlled evaluation of oral screen effects on intraoral pressure curve characteristics". European Journal of Orthodontics 32.5 (2010): 535-541.

33. Cattoni DM., et al. "Characteristics of the stomatognathic system of mouth breathing children: anthroposcopic approach". Pro Fono 9 (2007): 347-351.

34. Bhayya DP and Shyagali TR. "Prevalence of oral habits in 11-13year-old school children in Gulbarga city, India”. Virtual Journal of Orthodontics 8.3 (2009): 1-4.

35. Motta LJ., et al. "Association between halitosis and mouth breathing in children". Clinics 66.6 (2011): 939-942.

36. Guaba K., et al. "Prevalence of malocclusion and abnormal habits in North Indian rural children". Journal of Indian Society of Pedodontics and Preventive Dentistry 16 (1998): 26-30.

37. Kharbanda OP., et al. "Oral habits in school going children of Delhi: a prevalence study". Journal of Indian Society of Pedodontics and Preventive Dentistry 21 (2003): 120-124.

38. Conti PB., et al. "Assessment of the body posture of mouthbreathing children and adolescents". Jornal de Pediatria 87.4 (2011): 357-363.

39. Bresolin D., et al. "Facial characteristics of children who breathe through the mouth". Pediatrics 73 (1984): 622-625.
40. Trask GM., et al. "The effects of perennial allergic rhinitis on dental and skeletal development: a comparison of sibling pairs". American Journal of Orthodontics and Dentofacial Orthopedics 92 (1987): 286-293.

\section{Assets from publication with us}

- Prompt Acknowledgement after receiving the article

- Thorough Double blinded peer review

- Rapid Publication

- Issue of Publication Certificate

- High visibility of your Published work

Website: www.actascientific.com/

Submit Article: www.actascientific.com/submission.php Email us: editor@actascientific.com

Contact us: +919182824667 